\section{Fatores de risco cardiovascular em crianças de 7 a 10 anos de área urbana, Vitória, Espírito Santo, Brasil}

\author{
Cardiovascular risk factors in 7-to-10-year-old \\ children in Vitória, Espírito Santo State, Brazil
}

\begin{abstract}
The study aimed to identify the simultaneous occurrence of cardiovascular risk factors in children 7 to 10 years of age in Vitória, Espírito Santo State, Brazil, and investigate associated socioeconomic variables. In a sample of 1,282 children, anthropometric data were obtained and blood pressure was measured with an automatic device. Socioeconomic, nutritional, and physical activity data were obtained with a questionnaire. To evaluate the simultaneous occurrence of cardiovascular risk factors, an index was developed including overweight, high blood pressure, poor eating, and sedentary leisure $\geq 4$ hours/day. The simultaneous presence of four cardiovascular risk factors was defined as high risk. Socioeconomic status and maternal schooling were included in the logistic regression model. Some $34 \%$ of the children presented two cardiovascular risk factors, $20 \%$ three factors, and $6.4 \%$ four cardiovascular risk factors. Maternal schooling remained associated with high cardiovascular risk (OR: 7.36, 95\%CI: 2.09-25.97) and medium risk (OR: 2.57, 95\%CI: 1.58-4.20). Low maternal schooling was the most important factor associated with cardiovascular risk.
\end{abstract}

Child Health; Anthropometry; Hypertension; Overweight

\author{
Maria del Carmen Bisi Molina 1 \\ Carolina Perim de Faria 1 \\ Maria Pilar Montero 2 \\ Nágela Valadão Cade 1 \\ José Geraldo Mill 1
}

\section{Introdução}

As doenças do aparelho circulatório constituem a primeira causa de morte no Brasil há mais de três décadas. Embora conhecidos alguns de seus fatores de risco, a redução da morbi-mortalidade cardiovascular não tem sido uma tarefa fácil, tendo em vista a sua complexidade e a necessidade de iniciar precocemente o controle da hipertensão arterial, do tabagismo, da hipercolesterolemia e da obesidade 1 . Além desses, a Organização Mundial da Saúde (OMS) propõe a redução e o controle de outros fatores, como o alto consumo de álcool, a inatividade física e a dieta inadequada, dentro de uma abordagem integrada e em todas as faixas etárias ${ }^{2}$.

Os fatores de risco cardiovascular, tais como o excesso de peso, a hipertensão arterial, as dislipidemias, dentre outros, estão presentes em grande proporção em idosos 3 , trabalhadores $4 \mathrm{e}$ indivíduos em maior risco social 5. A ocorrência desses fatores em adolescentes também já vem sendo descrita 6 , porém há evidências de que o processo aterosclerótico possa se iniciar ainda mais cedo 7, aumentando progressivamente com a idade e com gravidade diretamente proporcional ao número de fatores de risco apresentados pelo indivíduo ${ }^{8}$.

Dentre os fatores de risco cardiovascular identificados em crianças brasileiras, o excesso de peso tem sido descrito com maior freqüência 9,10 . Ainda que baixo o porcentual de crianças 
que já foram submetidas à medida da pressão arterial 11, a elevação desta já vem sendo observada também em estudos localizados 12,13. Estudar os comportamentos de risco para as doenças cardiovasculares, tais como a inatividade física e a qualidade da alimentação, também é de fundamental importância para a compreensão dos diferentes contextos aos quais as crianças estão submetidas, embora de grande dificuldade operacional em investigações com esta faixa etária. Tais fatores foram considerados de risco para as doenças cardiovasculares em estudos com adolescentes 6,14, o que pode indicar a importância desses mesmos indicadores para outras faixas etárias.

Assim sendo, a identificação precoce de fatores de risco cardiovascular é de fundamental importância para que possam ser realizadas ações de promoção da saúde e o monitoramento em crianças brasileiras. Estudo com esse objetivo já foi realizado em outra cidade brasileira 12 , porém esta investigação é a primeira na cidade de Vitória, Espírito Santo, cujo resultado contribuirá sobremaneira para o maior conhecimento da situação epidemiológica neste grupo etário e para a tomada de decisões tanto na área da saúde como na da educação. Portanto, o objetivo deste estudo foi identificar a ocorrência simultânea de fatores de risco cardiovascular em amostra representativa de crianças de 7 a 10 anos de idade, domiciliadas na cidade de Vitória e investigar os fatores socioeconômicos associados.

\section{Material e métodos}

Estudo seccional de escolares do primeiro ciclo do ensino fundamental nas escolas do município (cobertura de 99\%). De um universo de aproximadamente 18.500 alunos, distribuídos em 105 escolas de Vitória, foi selecionada uma amostra aleatória por conglomerados em dois estágios, sendo a escola a unidade primária e a turma a unidade secundária. Os dados foram estratificados por cotas de tipo de escola (pública e particular), sexo (masculino e feminino) e por idade (7, 8, 9 e 10 anos). Foi definido um número de 40 crianças por escola pelo critério do número ótimo 15 , que minimiza custos do acesso direto da criança em relação ao acesso na escola e considera a correlação intraclasse dos alunos da mesma escola.

Foram excluídas do estudo as crianças com idade superior a 11 e inferior a 7 anos no momento da aferição das medidas na escola. A participação das crianças no estudo foi condicionada à assinatura do Termo de Consentimento Livre e Esclarecido por seus pais ou responsável, antes da realização das medidas antropométricas e hemodinâmicas.

A coleta de dados antropométricos (peso e estatura) foi realizada por estudantes de enfermagem devidamente treinados, conforme técnicas padronizadas 16. Buscando controlar erros de medida e/ou leitura de equipamentos e padronização de técnicas, foi realizado novo treinamento com base em estudo de variabilidade de medidas intra e interavaliadores. Foi feito um estudo piloto com 49 crianças em uma escola municipal experimental localizada no campus da Universidade Federal do Espírito Santo. Foram coletados dados de peso corporal em kg, precisão de 100g, em balanças modelo Family BWF (Tanita Corp., Arlington Heights, Estados Unidos). A estatura foi aferida em cm, precisão de $1 \mathrm{~mm}$, em estadiômetro portátil da marca Seca modelo 206 (Seca Ltd., Birmingham, Reino Unido) afixado à parede lisa e sem rodapé. $\mathrm{O}$ excesso de peso foi determinado de acordo com Cole et al. 17 para o índice de massa corpórea (IMC) e, neste estudo, as crianças foram classificadas em apenas dois grupos presença ou não de excesso de peso (IMC acima do percentil 85).

A pressão arterial foi verificada pelo método oscilométrico com aparelho automático marca OMRON (OMRON Eletrônica do Brasil, São Paulo, Brasil), modelo HEM-705CP, validado para pesquisa 18 . Foram utilizados manguitos OMRON de tamanho apropriado à circunferência dos braços das crianças - tamanho infantil para circunferência de 16 a $22 \mathrm{~cm}$ e tamanho adulto para circunferência de 23 a $33 \mathrm{~cm}$ - e desenvolvido um protocolo para verificação da pressão arterial que considerou as médias de duas medidas de pressão arterial sistólica (PAS) e diastólica (PAD) aferidas na criança, no período escolar e após 5 minutos de descanso. No caso de a diferença entre a 1a e a $2 \underline{a}$ medidas da PAS ou PAD ser maior que $5 \mathrm{mmHg}$ foi realizada uma 3a medida e considerada a média entre a 2 a e a 3a medidas da PAS e PAD. Para a classificação da pressão foram consideradas as curvas para determinação do percentil da estatura da criança de acordo com a idade e o sexo, segundo o National High Blood Pressure Education Program dos Estados Unidos (NHBPEP) 19 e a tabela de percentil da pressão arterial referenciada pela $V$ Diretrizes de Hipertensão Arterial 20. As crianças com PAS e/ou PAD igual ou acima do percentil 95 para sexo, idade e estatura foram classificadas na condição de pressão arterial elevada. Todas as medidas foram realizadas nas dependências das escolas visitadas.

Dados socioeconômicos, seguindo o Critério de Classificação Econômica Brasil 21, e de atividade física da criança foram obtidos a partir 
de questões específicas inseridas em questionário estruturado, baseado em estudo com adolescentes 22, e as informações sobre consumo alimentar das crianças foram obtidas valendose de um questionário de freqüência alimentar (QFA), preenchido por seus pais ou responsável pela criança. A seleção dos itens alimentares foi baseada em estudo com crianças em São Paulo 9 e em outro com adolescentes no Rio de Janeiro 23 , e incorporado em item à parte a freqüência de consumo de peixe, por se tratar de um alimento típico da região. Concomitantemente à coleta dos dados na amostra foi realizado um estudo de reprodutibilidade com uma subamostra de 91 indivíduos. Para a maioria dos alimentos do QFA foi obtida uma concordância moderada $(0,6-0,8)$ ou muito boa $(>0,8)$. Os alimentos que não obtiveram concordância igual ou acima de 0,6 não foram computados para fins de análise, e os itens "arroz" e "carne de boi/frango" não foram incluídos nesta proposta por não serem discriminantes da alimentação habitual desse grupo. Assim, o QFA avaliou o consumo de 15 itens alimentares em três categorias. Da mesma forma, foi realizado um estudo de reprodutibilidade do questionário de atividade física e obtidos valores entre 0,6 e 0,98.

Foram investigados os seguintes fatores de risco cardiovascular, levando-se em consideração evidências encontradas em estudos com crianças e adolescentes: (i) excesso de peso; (ii) pressão arterial elevada em medida única; (iii) tempo diário de lazer sedentário igual ou superior a 4 horas diárias; e (iv) alimentação de baixa qualidade.

Para obtenção da variável atividade de lazer sedentária foram somados os tempos utilizados diariamente em uso de videogame, computador e televisão. Para avaliação da alimentação foi desenvolvido um índice de qualidade da alimentação do escolar (Índice ALES) com base na freqüência de consumo dos 15 itens alimentares (fruta, suco natural, legume, verdura crua, feijão, leite, peixe, doces e balas, biscoito recheado e chips, refrigerante, hambúrguer, salgado frito, batata e aipim frito, maionese, macarrão instantâneo) e o hábito de fazer a primeira refeição matinal (desjejum), dando a cada freqüência específica uma pontuação. Os valores das freqüências individuais foram somados e a partir dos valores dos tercis de distribuição, isto é, 3 e 6, foram definidas três categorias de qualidade da alimentação: < 3 (baixa qualidade), entre 3-6 (qualidade intermediária) e $\geq 6$ (boa qualidade).

As crianças foram classificadas em três grupos, conforme o número de fatores presentes, a saber: nenhum ou apenas 1 fator presente; $2 \mathrm{ou}$ 3 fatores presentes; e 4 fatores presentes. Para o estudo dos fatores associados ao número de fatores de risco cardiovascular foram investigadas as seguintes variáveis: sexo (masculino e feminino); idade da criança (7, 8, 9 ou 10 anos); classe socioeconômica $(A+B, C, D+E)$, baseada na escolaridade do chefe da família e posse de bens; e escolaridade materna ( $\leq 3$ anos de estudo, 4 a 10 anos de estudo, 10 a 14 anos de estudo e igual ou superior a 15 anos de estudo).

As variáveis qualitativas foram apresentadas em porcentuais e utilizado o teste do qui-quadrado $\left(\chi^{2}\right)$ para verificar a hipótese de homogeneidade de proporções. Para a análise multivariada foram utilizados procedimentos de regressão logística multinomial, sendo a variável dependente o número de fatores de risco cardiovascular, cuja condição identificada como "nenhum ou um fator de risco cardiovascular" a categoria de referência. O nível de significância para inclusão de variáveis foi estabelecido em $10 \%$, utilizado para qualquer uma das categorias da variávelresposta. As análises estatísticas foram realizadas utilizando-se o programa SPSS for Windows, versão 17 (SPSS Inc., Chicago, Estados Unidos). Foram estimadas as razões de produtos cruzados odds ratio (OR) ajustadas e os respectivos intervalos de $95 \%$ de confiança para as variáveis que permaneceram no modelo. O presente estudo foi aprovado pelo Comitê de Ética em Pesquisa do Centro Biomédico da Universidade Federal do Espírito Santo em 26 de outubro de 2006, sob o número de registro 089/06.

\section{Resultados}

Das 1.637 famílias que autorizaram a participação de seus filhos nesta pesquisa, 336 (20,5\%) não realizaram a segunda etapa do estudo (preenchimento do questionário enviado para os domicílios) e 19 questionários foram excluídos na etapa de controle de qualidade. Assim sendo, foram obtidos e avaliados dados de 1.282 crianças (538 meninos e 744 meninas) matriculadas em 26 escolas públicas e 6 privadas.

A distribuição da amostra segundo variáveis sociodemográficas por sexo pode ser observada na Tabela 1. Aproximadamente $28 \%$ das mães das crianças estudaram menos de 8 anos, $20 \%$ de 8 a 10 anos, $36 \%$ de 11 a 14, e $17 \%$ relataram 15 ou mais anos de estudo. Quanto à classificação socioeconômica, foram obtidos os seguintes percentuais: classes $A+B, 23,3 \%$; classe C, 35,1; e classes D + E, 41,6\%. A proporção de crianças com 7, 8, 9 e 10 anos foi de 19,7\%, 28\%, 28,1\% e $24,3 \%$, respectivamente. Não foram encontradas diferenças significativas entre as variáveis sociodemográficas estudadas e sexo. 
No que diz respeito aos fatores de risco cardiovascular estudados, foram encontradas as seguintes prevalências: $23,2 \%$ de excesso de peso, $13,8 \%$ de pressão arterial elevada, $48,7 \%$ com 4 ou mais horas de lazer sedentário e 40,6\% com alimentação de baixa qualidade. Observa-se diferença significativa apenas entre lazer sedentário igual ou superior a 4 horas diárias e sexo (Tabela 2).

Em aproximadamente $39 \%$ das crianças estudadas não foi observado nenhum fator de risco cardiovascular ou apenas um fator, sendo o mais freqüente a atividade de lazer sedentária maior ou igual a 4 horas diárias. Mais de $50 \%$ das crianças estudadas apresentaram 2 ou 3 fatores de risco e em $6,4 \%$ da amostra foram observados os quatro fatores de risco estudados, isto é, presença de excesso de peso, pressão arterial elevada, alimentação de baixa qualidade e 4 ou mais horas de lazer sedentário diário.

Na Tabela 3 pode ser observada a classificação adotada com base no número de fatores de risco cardiovascular, segundo variáveis demográficas e socioeconômicas. O percentual de crianças com quatro fatores de risco cardiovascular é maior na classe socioeconômica $\mathrm{C}$ e no grupo de crianças cuja escolaridade materna é mais baixa ( $\mathrm{p}<$ 0,000 ). Não foi observada diferença significativa

Tabela 1

Distribuição da amostra segundo variáveis sociodemográficas por sexo. Vitória, Espírito Santo, Brasil, 2007.

\begin{tabular}{|c|c|c|c|c|c|c|c|}
\hline \multirow[t]{2}{*}{ Variável } & \multicolumn{2}{|c|}{ Masculino } & \multicolumn{2}{|c|}{ Feminino } & \multirow{2}{*}{$\begin{array}{l}\text { Valor } \\
\text { de p }\end{array}$} & \multicolumn{2}{|c|}{ Total } \\
\hline & $\mathbf{n}$ & $\%$ & $\mathrm{n}$ & $\%$ & & $\mathrm{n}$ & $\%$ \\
\hline \multicolumn{8}{|c|}{ Escolaridade materna (em anos) } \\
\hline$<8$ & 144 & 26,8 & 212 & 28,5 & 0,720 & 356 & 27,8 \\
\hline $8-10$ & 106 & 19,7 & 145 & 19,5 & & 251 & 19,6 \\
\hline $11-14$ & 189 & 35,1 & 267 & 35,9 & & 456 & 35,6 \\
\hline$\geq 15$ & 99 & 18,4 & 120 & 16,1 & & 219 & 17,1 \\
\hline \multicolumn{8}{|c|}{ Classe socioeconômica } \\
\hline$A+B$ & 113 & 23,8 & 149 & 23,0 & 0,854 & 262 & 23,3 \\
\hline C & 162 & 34,2 & 232 & 35,7 & & 394 & 35,1 \\
\hline$D+E$ & 199 & 42,0 & 268 & 41,3 & & 467 & 41,6 \\
\hline \multicolumn{8}{|c|}{ Idade (em anos) } \\
\hline 7 & 103 & 19,1 & 149 & 20,0 & 0,183 & 252 & 19,7 \\
\hline 8 & 139 & 25,8 & 220 & 29,6 & & 359 & 28,0 \\
\hline 9 & 150 & 27,9 & 210 & 28,2 & & 360 & 28,1 \\
\hline 10 & 146 & 27,1 & 165 & 22,2 & & 311 & 24,3 \\
\hline
\end{tabular}

Tabela 2

Prevalência de fatores de risco cardiovascular entre crianças de 7 a 10 anos de idade, segundo sexo. Vitória, Espírito Santo, Brasil, 2007.

\begin{tabular}{|c|c|c|c|c|c|c|c|}
\hline \multirow[t]{2}{*}{ Fatores de risco cardiovascular } & \multicolumn{2}{|c|}{ Masculino } & \multicolumn{2}{|c|}{ Feminino } & \multicolumn{2}{|c|}{ Total } & \multirow{2}{*}{$\begin{array}{l}\text { Valor } \\
\text { de } p \text { * }\end{array}$} \\
\hline & $\mathbf{n}$ & $\%$ & $\mathbf{n}$ & $\%$ & $\mathbf{n}$ & $\%$ & \\
\hline Excesso de peso & 123 & 22,9 & 175 & 23,5 & 298 & 23,2 & 0,783 \\
\hline Pressão arterial elevada ** & 63 & 12,9 & 96 & 13,9 & 159 & 13,8 & 0,607 \\
\hline Lazer sedentário $\geq 4$ horas diárias *** & 266 & 53,8 & 301 & 45,0 & 566 & 48,7 & 0,002 \\
\hline Alimentação de baixa qualidade & 203 & 37,7 & 318 & 42,7 & 521 & 40,6 & 0,072 \\
\hline
\end{tabular}

* Teste do qui-quadrado;

** $\mathrm{n}=1.181$;

$\star \star \star n=1.152$ 
Distribuição percentual do número de fatores de risco cardiovascular em crianças de 7 a 10 anos de idade, segundo variáveis demográficas e socioeconômicas. Vitória, Espírito Santo, Brasil, 2007.

\begin{tabular}{|c|c|c|c|c|}
\hline \multirow[t]{2}{*}{ Variável } & \multicolumn{4}{|c|}{ Número de fatores de risco cardiovascular (\%) } \\
\hline & 0 ou 1 & 2 ou 3 & 4 & Valor de $\mathrm{p}$ \\
\hline Escolaridade materna (em anos) & & & & $<0,000$ \\
\hline$\leq 3$ & 30,6 & 62,4 & 7,0 & \\
\hline 4-10 & 36,7 & 55,0 & 8,4 & \\
\hline $11-14$ & 37,9 & 55,3 & 6,8 & \\
\hline$\geq 15$ & 59,4 & 38,4 & 2,3 & \\
\hline Classe socioeconômica & & & & $<0,000$ \\
\hline$A+B$ & 54,6 & 41,2 & 4,2 & \\
\hline C & 38,3 & 54,3 & 7,4 & \\
\hline$D+E$ & 33,8 & 59,3 & 6,9 & \\
\hline Idade (em anos) & & & & 0,178 \\
\hline 7 & 43,7 & 48,8 & 7,5 & \\
\hline 8 & 40,1 & 53,8 & 6,1 & \\
\hline 9 & 40,8 & 53,6 & 5,6 & \\
\hline 10 & 33,1 & 60,1 & 6,8 & \\
\hline Sexo & & & & 0,198 \\
\hline Masculino & 39,1 & 55,9 & 5,0 & \\
\hline Feminino & 39,5 & 53,1 & 7,4 & \\
\hline
\end{tabular}

entre número de fatores de risco cardiovascular e as variáveis sexo e idade.

Observa-se na Tabela 4 os resultados da análise de regressão multinomial. Foram incluídas no modelo as variáveis classe socioeconômica e escolaridade materna, porém a que se manteve associada ao risco cardiovascular médio e alto foi a escolaridade materna. Cabe destacar que o fato de a mãe ser analfabeta ou não ter completado o ensino fundamental eleva aproximadamente 7,4 vezes a chance de a criança apresentar quatro fatores de risco cardiovascular, e esta chance vai diminuindo com o aumento da escolaridade materna.

\section{Discussão}

Informações sobre a presença simultânea de fatores de risco cardiovascular em crianças pode ser útil para a identificação de subgrupos populacionais que deveriam receber maior atenção dos serviços de saúde em programas de prevenção, como é o caso dos que apresentaram os quatro fatores de risco estudados nesta pesquisa.

Este estudo contempla fatores reconhecidamente de risco para as doenças cardiovasculares em adultos e crianças, ainda que não incorpo- rado nenhum indicador de dislipidemia, tendo em vista a sua importância para avaliação da progressão da aterosclerose desde a infância. A maioria dos estudos epidemiológicos realizados no Brasil sobre a ocorrência de hipercolesterolemia foi desenvolvida em amostra de crianças e adolescentes, o que dificulta a interpretação dos achados para a faixa etária de 7 a 10 anos, porém parece haver uma relação estreita com o índice de massa corporal elevado em crianças 24 .

$\mathrm{O}$ fator de risco cardiovascular de menor prevalência encontrado na amostra foi a presença de pressão arterial elevada, porém se levarmos em consideração que o grupo estudado é de crianças de 7 a 10 anos, pode-se inferir que é elevado o número de indivíduos que deveriam ser acompanhados sistematicamente pelos serviços de saúde. Considerando que a hipertensão arterial é o preditor mais importante da mortalidade cardiovascular e que as desigualdades sociais presentes determinam a sua precocidade 25 , é importante a detecção precoce da alteração da pressão arterial em crianças cujas famílias se encontram em maior risco social.

O tempo médio utilizado pelas crianças estudadas assistindo à televisão alcançou aproximadamente 3,3 horas por dia, enquanto o tempo médio em atividades de lazer sedentário, 
Fatores associados ao risco cardiovascular em crianças de 7 a 10 anos: razão de chances (RC) brutas e ajustadas com respectivos intervalos de confiança. Vitória, Espírito Santo, Brasil, 2007.

\begin{tabular}{|c|c|c|c|c|}
\hline \multirow[t]{3}{*}{ Variáveis } & \multicolumn{4}{|c|}{ Número de fatores de risco cardiovascular } \\
\hline & \multicolumn{2}{|c|}{2 ou 3} & \multicolumn{2}{|c|}{4} \\
\hline & Sem ajuste & Ajustada * & Sem ajuste & Ajustada * \\
\hline \multicolumn{5}{|l|}{ Escolaridade da mãe } \\
\hline Fundamental incompleto & $3,15(2,20-4,51)$ & $2,57(1,58-4,20)$ & $5,96(2,21-16,10)$ & $7,36(2,09-25,97)$ \\
\hline Fundamental completo & $2,32(1,59-3,39)$ & $1,73(1,05-2,73)$ & $5,93(2,16-16,31)$ & $6,99(1,99-29,52)$ \\
\hline Ensino Médio & $2,25(1,61-3,15)$ & $1,82(1,20-2,78)$ & $4,66(1,76-12,31)$ & $4,92(1,52-15,96)$ \\
\hline Ensino Superior & 1,00 & 1,00 & 1,00 & 1,00 \\
\hline \multicolumn{5}{|l|}{ Classe socioeconômica } \\
\hline$A+B$ & $0,43(0,31-0,59)$ & $0,71(0,46-1,07)$ & $0,38(0,18-0,78)$ & $1,03(0,44-2,41)$ \\
\hline C & $0,81(0,61-1,08)$ & $0,94(0,69-1,27)$ & $0,95(0,55-1,64)$ & $1,19(0,66-2,13)$ \\
\hline $\mathrm{D}+\mathrm{E}$ & 1,00 & 1,00 & 1,00 & 1,00 \\
\hline
\end{tabular}

* As variáveis foram ajustadas entre si.

comumente referido como uma variação de seu componente mais importante - a televisão - aproximou-se de quatro horas diárias. Silva \& Malina 26, em estudo com adolescentes, encontraram valores ainda maiores, e Mondini et al. ${ }^{9}$ observaram que $84,4 \%$ das crianças estudadas em Cajamar, São Paulo, assistem à televisão por mais de duas horas por dia. Provavelmente, esses resultados refletem o menor envolvimento das crianças em atividades educacionais extraclasses, a conscientização dos pais quanto à necessidade de restringir o tempo de lazer sedentário de seus filhos ou, até mesmo, uma dificuldade de informar adequadamente as atividades de seus filhos. Cerca de 8\% das mães de crianças estudadas não responderam a essas questões, porém esse fato não estava associado ao baixo nível de escolaridade, após análise dos que não tinham todos os seus dados disponíveis.

A prevalência encontrada de excesso de peso na amostra foi de $23,2 \%$, valor considerado mediano, uma vez que estudos mais recentes encontraram valores ainda mais altos como os de Costa et al. 10 e Brasil et al. 27. É importante ressaltar que a metodologia para a definição de excesso de peso disponível na literatura não é a mesma, portanto, a comparação de resultados deve ser feita com cautela. Ainda assim, prevalências de sobrepeso na casa dos $20-25 \%$ são indicadores claros da evolução do excesso de peso para a faixa etária estudada no Brasil. Estudo baseado no National Health and Nutrition Examination Survey (NHANES) de 2002-2003 apresentou prevalência de sobrepeso em torno de $18,8 \%$ para crianças americanas entre 6 e 11 anos 28. Os valores encontrados são expressivos e denotam o rápido crescimento das prevalências de sobrepeso e obesidade, elevando o risco para as dislipidemias e, por conseguinte, para as doenças cardiovasculares.

Aspecto de grande importância na ocorrência de doenças cardiovasculares está relacionado aos hábitos alimentares desenvolvidos desde a infância. Observamos nesta amostra porcentual elevado de crianças que realizam uma alimentação de baixa qualidade, como por exemplo: menos de uma porção diária de frutas, hortaliças, feijão e leite, além da omissão do desjejum e uso diário de biscoitos ou frituras. $\mathrm{O}$ uso de um índice para avaliação da qualidade da dieta pode apresentar vantagem em relação ao uso de apenas um grupo de alimentos para avaliação de uma alimentação saudável. Ainda que bem consolidada a relação entre alimentação e doenças crônicas 29 , provavelmente o efeito da dieta sobre o aumento da prevalência de fatores de risco cardiovascular não seja tão evidente na infância, porém deve ser incluído em estudos que visam a quantificar o aumento da probabilidade de desenvolvimento de doenças cardiovasculares, pois já é conhecida a associação do consumo de determinados nutrientes sobre o aumento da pressão arterial, bem como a omissão do desjejum sobre a ocorrência de obesidade em crianças 30 . Os resultados deste estudo coincidem com os encontrados por Gama et al. 13 em crianças de 6 a 9 anos de idade de área de baixa renda do Rio de Janeiro, onde a prevalência de fatores de risco cardiovascular foi elevada. 
Conclui-se neste estudo que os fatores de risco cardiovascular estão presentes em parcela significativa das crianças estudadas e que há uma aglomeração maior destes fatores em crianças cujas mães apresentavam menor escolaridade. Situação semelhante foi encontrada em população adulta domiciliada na cidade do Rio de Janeiro 5 , demonstrando que a escolaridade é de fato um dos mais importantes determinantes socioeconômicos associados ao risco cardiovascular tanto em adultos como em crianças. Provavelmente, a baixa escolaridade materna influi em diversos aspectos da vida da criança, tais como a maior exposição ao sedentarismo e à alimentação de baixa qualidade, seja por menor acesso a alimentos saudáveis e informações adequadas e/ou pela menor capacidade de discernir entre o que é considerado de fato saudável. Medidas de controle e prevenção dos fatores de risco cardiovascular devem ser tomadas precocemente em todos os níveis da atenção à saúde e em diferentes áreas, a fim de contribuir para a redução das iniqüidades em saúde e na melhoria da qualidade de vida.

\section{Resumo}

O objetivo do estudo foi identificar a ocorrência simultânea de fatores de risco cardiovascular em crianças de 7 a 10 anos de Vitória, Espírito Santo, Brasil, e investigar variáveis socioeconômicas associadas. Foram pesquisadas 1.282 crianças e obtidos dados antropométricos e medidas da pressão arterial com aparelho automático. Dados socioeconômicos, de alimentação e de atividade física foram obtidos com base em questionário. Para avaliação da ocorrência simultânea de fatores de risco cardiovascular foi desenvolvido um índice a partir da presença de excesso de peso, pressão arterial elevada, alimentação de bai$x a$ qualidade e lazer sedentário $\geq 4$ h/dia. Presença simultânea de quatro fatores de risco cardiovascular foi identificada como risco alto. Classe socioeconômica e escolaridade materna foram incluídas no modelo de regressão logística. Cerca de $34 \%$ das crianças apresentaram dois fatores de risco cardiovascular, 20\% três fatores e 6,4 quatro fatores de risco cardiovascular. A escolaridade materna se manteve associada ao risco cardiovascular alto (OR: 7,36, IC95\%: 2,09-25,97) $e$ médio (OR: 2,57, IC95\%: 1,58-4,20). Baixa escolaridade materna foi o fator mais importante associado ao risco cardiovascular.

Saúde da Criança; Antropometria; Hipertensão; Sobrepeso 


\section{Colaboradores}

M. C. B. Molina contribuiu com a elaboração do projeto, análise e interpretação dos dados, concepção e redação do artigo. C. P. Faria participou da elaboração do projeto, coordenação do trabalho de campo e redação do artigo. M. P. Montero participou da concepção do artigo e da análise dos dados. N. V. Cade e J. G. Mill contribuíram com a interpretação dos resultados e a revisão crítica do artigo.

\section{Agradecimentos}

Financiado pelo Fundo de Apoio à Ciência e Tecnologia (FACITEC-Vitória - Edital Educação). Aos diretores e professores das escolas participantes do estudo pela colaboração na fase de coleta de dados.

\section{Referências}

1. Kumanyika SK, Obarzanek E, Stettler N, Bell R, Field AE, Fortmann SP, Franklin BA, et al. Population-based prevention of obesity: the need for comprehensive promotion of healthful eating, physical activity, and energy balance. A scientific statement from American Heart Association Council on Epidemiology and Prevention, Interdisciplinary Committee for Prevention (formerly the Expert Panel on Population and Prevention Science). Circulation 2008; 118:428-64.

2. World Health Organization. The World health report 2002: reducing risks, promoting health life. http://www.who.int/whr/2002/media_centre/en/ index.html (acessado em 05/Fev/2009).

3. Pereira JC, Barreto SM, Passos VMA. O perfil de saúde cardiovascular dos idosos brasileiros precisa melhorar: estudo de base populacional. Arq Bras Cardiol 2008; 91:1-10.

4. Cassani RSL, Nobre F, Pazin Filho A, Schmidt A. Prevalência de fatores de risco cardiovascular em trabalhadores de uma indústria brasileira. Arq Bras Cardiol 2009; 92:16-22.
5. Marins VM, Almeida RM, Pereira RA, Sichieri R. The association between socioeconomic indicators and cardiovascular disease risk factors in Rio de Janeiro, Brazil. J Biosoc Sci 2007; 39:221-9.

6. Romanzini M, Reichert FF, Lopes AS, Petroski EL, Farias Júnior JC. Prevalência de fatores de risco cardiovascular em adolescentes. Cad Saúde Pública 2008; 24:2573-81.

7. Boyd S, Koenigsberg J, Falkner B, Gidding S, Hassink S. Effect of obesity and high blood pressure on plasma lipid levels in children and adolescents. Pediatrics 2005; 116:442-6.

8. Sociedade Brasileira de Cardiologia, Departamento de Aterosclerose. I diretriz de prevenção da aterosclerose na infância e na adolescência. Arq Bras Cardiol 2005; 85:3-36.

9. Mondini L, Levy RB, Saldiva SRDM, Venâncio SI, Aguiar JA, Stefanini MLR. Prevalência de sobrepeso e fatores associados em crianças ingressantes no ensino fundamental em um município da região metropolitana de São Paulo, Brasil. Cad Saúde Pública 2007; 23:1825-34. 
10. Costa RF, Cintra IP, Fisberg M. Prevalência de sobrepeso e obesidade em escolares da Cidade de Santos, SP. Arq Bras Endocrinol Metab 2006; 50:60-7.

11. Silva MAM, Rivera IR, Souza MGB, Carvalho ACC. Medida da pressão arterial em crianças e adolescentes: recomendações das diretrizes de hipertensão arterial e prática médica atual. Arq Bras Cardiol 2007; 88:491-5.

12. Silva MAM, Rivera IR, Ferraz MRMT, Pinheiro AJT, Alves SWS, Moura AA, et al. Prevalência de fatores de risco cardiovascular em crianças e adolescentes da rede de ensino da Cidade de Maceió. Arq Bras Cardiol 2005; 84:387-92.

13. Gama SR, Carvalho MS, Chaves CRMM. Prevalência em crianças de fatores de risco para as doenças cardiovasculares. Cad Saúde Pública 2007; 23:2239-45.

14. Bas M, Altan T, Dincer D, Aran E, Kaya HG, Yüksek O. Determination of dietary habits as a risk factor of cardiovascular heart disease in Turkish adolescents. Eur J Nutr 2005; 44:174-82.

15. Silva NN. Amostragem probabilística. São Paulo: Edusp; 1998.

16. World Health Organization. Physical status: the use and interpretation of anthropometry indicators of nutritional status. Geneva: World Health Organization; 1995. (Technical Report Series, 854).

17. Cole TJ, Bellizzi MC, Flegal KM, Dietz WH. Establishing a standard definition for child overweight and obesity worldwide: international survey. BMJ 2000; 320:1240-3.

18. White WB, Anwar YA. The importance of clinical validation of semi-automatic and automatic blood pressure monitors in adults according to national standards or guidelines. Blood Press Monit 1998; 3 Suppl 1:S7-10.

19. National High Blood Pressure Education Program Working Group on High Blood Pressure in Children and Adolescents. The fourth report on the diagnosis, evaluation, and treatment of high blood pressure in children and adolescents. Pediatrics 2004; 114:555-76.

20. Sociedade Brasileira de Cardiologia/Sociedade Brasileira de Hipertensão/Sociedade Brasileira de Nefrologia. V diretrizes brasileiras de hipertensão arterial. http://departamentos.cardiol.br/dha/vdi retriz/vdiretriz.asp (acessado em 20/Mai/2007).
21. Associação Brasileira de Institutos de Pesquisa e Mercado. Critério de classificação socioeconômica: critério ABIPEME. Reformulação do critério anterior. http://www.anep.org.br/mural/anep/ (acessado em 12/Mar/2007).

22. Hallal PC, Bertoldi AD, Gonçalves H, Victora CG. Prevalência de sedentarismo e fatores associados em adolescentes de 10-12 anos de idade. Cad Saúde Pública 2006; 22:1277-87.

23. Castro IRR, Cardoso LO, Engstrom EM, Levy RB, Monteiro CA. Vigilância de fatores de risco para doenças não transmissíveis entre adolescentes: a experiência da Cidade do Rio de Janeiro, Brasil. Cad Saúde Pública 2008; 24:2279-88.

24. Coronelli CLS, Moura EC. Hipercolesterolemia em escolares e seus fatores de risco. Rev Saúde Pública 2003; 37:24-31.

25. Ishitani LH, Franco GC, Perpetuo IHO, França E. Desigualdade social e mortalidade precoce por doenças cardiovasculares no Brasil. Rev Saúde Pública 2006; 40:684-91.

26. Silva RCR, Malina RM. Nível de atividade física em adolescentes do Município de Niterói, Rio de Janeiro, Brasil. Cad Saúde Pública 2000; 16:1091-7.

27. Brasil L, Fisberg M, Maranhão HS. Excesso de peso de escolares em região do Nordeste Brasileiro: contraste entre as redes de ensino pública e privada. Rev Bras Saúde Matern Infant 2007; 7:405-12.

28. Ogden CL, Carroll MD, Curtin LR, McDowell MA, Tabak CJ, Flegal KM. Prevalence of overweight and obesity in the United States, 1999-2004. JAMA 2006; 295:1549-55.

29. World Health Organization. Diet, nutrition and the prevention of chronic diseases. Report of a joint WHO/FAO expert consultation. Geneva: World Health Organization; 2003.

30. Kranz S, Findeis JL, Shrestha SS. Uso do Índice de Qualidade da Dieta Infantil Revisado para avaliar a dieta alimentar de pré-escolares, seus preditores sociodemográficos e sua associação com peso corporal. J Pediatr (Rio J.) 2008; 84:26-34.

Recebido em 13/Nov/2009

Versão final reapresentada em 17/Mar/2010

Aprovado em 29/Mar/2010 